\title{
USO DE BACTERIAS ÁCIDO LÁCTICAS PARA DESCONTAMINACIÓN DE ESTIÉRCOL PORCINO MEDIANTE ENSILAJE EXPERIMENTAL
}

\author{
José Martín RUVALCABA-GÓMEZ ${ }^{2.3}$, Ramón Ignacio ARTEAGA-GARIBAY ${ }^{1 *}$, \\ Gerardo DOMÍNGUEZ-ARAUJO ${ }^{3}$, Alberto Jorge GALINDO-BARBOZA ${ }^{3}$, \\ Gerardo SALAZAR-GUTIÉRREZ ${ }^{3}$, Marcos Daniel MARTÍNEZ-PEÑA ${ }^{1 \dagger} \mathrm{y}$ \\ Raúl Jacobo DELGADO-MACUIL ${ }^{2}$
}

${ }^{1}$ Centro Nacional de Recursos Genéticos. Instituto Nacional de Investigaciones Forestales, Agrícolas y Pecuarias. Boulevard de la Biodiversidad 400, Tepatitlán de Morelos, Jalisco, México. C.P. 47600

${ }^{2}$ Centro de Investigación en Biotecnología Aplicada. Instituto Politécnico Nacional. Ex-Hacienda San Juan Molino, Carretera Estatal Tecuexcomac-Tepetitla, km 1.5, Tlaxcala, México. C.P. 90700.

${ }^{3}$ Campo Experimental Centro Altos de Jalisco. Instituto Nacional de Investigaciones Forestales, Agrícolas y Pecuarias. Boulevard de la Biodiversidad 2470, Tepatitlán de Morelos, Jalisco, México, C.P. 47600.

${ }^{\dagger}$ Los autores informan del sensible fallecimiento de Marcos Daniel Martínez Peña, ocurrido poco antes de la publicación de este artículo.

*Autor de correspondencia: arteaga.ramon@inifap.gob.mx

(Recibido junio 2017; aceptado abril 2018)

Palabras clave: ensilaje, cerdaza, fermentación acido-láctica, descontaminación

\section{RESUMEN}

La actividad porcina genera diariamente una gran cantidad de residuos conformados por nutrientes y microorganismos que potencialmente contribuyen a la contaminación de agua, aire y suelos, y la subsecuente generación de olores desagradables. Diversas estrategias se han desarrollado para mitigar el efecto contaminante de las excretas porcinas y aprovechar sus nutrientes; tal es el caso del ensilaje, que consiste en la fermentación de las excretas. En este estudio se evaluó el efecto del ensilaje del estiércol porcino adicionado con dos fuentes diferentes de carbohidratos y dos fuentes de bacterias ácido lácticas, sobre la reducción de diferentes grupos de microorganismos. Durante el proceso se observó la disminución del pH (hasta 4.6) y la carga microbiana, sobre todo para organismos coliformes (reducción mayor a 5 logaritmos) y hongos y levaduras, así como la eliminación total del grupo de enterococos; mientras que el contenido de bacterias ácido lácticas, principales responsables de la fermentación, permaneció constante. Los mejores resultados se observaron mediante el uso de sorgo como fuente de carbohidratos en combinación con suero de leche, subproducto de la elaboración de queso, como fuente de bacterias ácido lácticas, tanto en disminución del $\mathrm{pH}$ como en la reducción de algunos indicadores microbiológicos, incluidas las bacterias productoras de ácido sulfhídrico. Mediante el ensilaje del estiércol porcino se favorece la disminución del $\mathrm{pH}$ por acción de las bacterias ácido lácticas a niveles que limitan la sobrevivencia de microorganismos asociados a contaminación fecal, y lo transforman en un producto seguro, desde la perspectiva microbiológica, para su manejo y posible aprovechamiento subsecuente.

Key words: silage, pig manure, lactic-acid fermentation, decontamination 


\begin{abstract}
Swine production generates every day a large amount of residues, including nutrients and microorganisms, which potentially contribute to contamination of air, water and soil, and generation of unpleasant odors. Many strategies have been developed in order to mitigate the contaminant effect of swine manure and to take advantage of its nutrients and beneficial compounds; silage is an example of these, and consists in swine manure fermentation mainly under anaerobic conditions. The aim of this study was to assess the effect of applying a process of silage to swine manure added with two different sources of carbohydrates and two different sources of lactic-acid bacteria, under the reduction of different microbiological indicators. Through the process, it was observed the reduction of $\mathrm{pH}$ levels (until reaching 4.6) and microbial content, mainly for coliforms (reduction greater than $5 \mathrm{log}$ ), molds and yeasts, and total elimination of enterococci. On the other hand, significant decline was not reported for the lactic-acid bacteria content, main responsible of fermentation process. Best results were observed through using sorghum as a source of carbohydrates and cheese whey as a source of lactic-acid bacteria, for both, decrease of $\mathrm{pH}$ and reduction of some microbiological indicators, including bacteria that produce sulfuric acid. Therefore, silage process, applied to swine manure, induces the decrease of $\mathrm{pH}$ for action of lactic-acid bacteria until limit the survival of bacteria associated with fecal contamination, making of swine a transformed and microbiologically safe residue for its handling and subsequent use.
\end{abstract}

\section{INTRODUCCIÓN}

La producción de carne de cerdo en México es de aproximadamente $1291000 \mathrm{t}$ al año y en su mayoría se concentra en los estados de Jalisco, Sonora, Guanajuato, Puebla, Yucatán y Michoacán (SIAP 2014).

Asociado a la producción de carne, la actividad porcina genera diariamente una cantidad importante de desechos en sus unidades de producción, principalmente estiércol, cuyo manejo representa un gran reto para los productores.

Se ha reportado que la excreción fecal en cerdos puede ser de hasta $1.35 \mathrm{~kg}$ de material fresco por cada $\mathrm{kg}$ de materia seca ingerida, con un promedio de $25 \%$ de contenido de materia seca y alrededor de $70 \%$ de materia orgánica (Martínez et al. 2004).

No obstante, la cantidad y composición del estiércol porcino dependerá de factores como el fin zootécnico, edad de los animales, tipo de alimentación y condiciones de confinamiento (Sánchez y González 2005).

Las excretas pueden contener una cantidad significativa de compuestos bioquímicos con posibles aplicaciones, incluida la nutrición animal (BorgesGómez et al. 2003, Bórquez et al. 2009, Bulkowska et al. 2012, Moset et al. 2014), que favorecen el mantenimiento y proliferación de una importante carga de microorganismos propios de este tipo de residuos.

La microbiota del estiércol incluye diversas especies, principalmente de los géneros Escherichia,
Enterococcus, Clostridium, Bacillus, Listeria, Salmonella, Campylobacter, algunos hongos y levaduras, e incluso algunos virus (Sahlström et al. 2008, Masse et al. 2011, Zhang et al. 2011).

La combinación de nutrientes y microorganismos en el estiércol porcino, además de su manejo deficiente, ha provocado que la producción de cerdos contribuya con hasta un $13 \%$ a la emisión de gases y compuestos de efecto invernadero (GyCEI) a nivel mundial (707 000 t de gases como $\mathrm{CO}_{2}, \mathrm{CH}_{4}$ y NO 2 ) (Steinfeld et al. 2006, FAO 2013a, FAO 2013b, Philippe y Nicks 2015).

En México se ha reportado que la actividad porcina genera alrededor de $16000 \mathrm{t}$ diarias de estiércol y la mayoría son arrojadas a tierras de cultivo, cauces de ríos y drenajes municipales (García et al. 2010), lo que contribuye a la contaminación de mantos acuíferos y otros recursos naturales.

Con el objetivo de mitigar el daño ambiental que puede provocar la excreta porcina, se han realizado diferentes estudios para evaluar los efectos del uso de procesos orientados a la descontaminación de este residuo.

Procesos como la fermentación, acidificación y pasteurización, han mostrado reducir la carga microbiana del estiércol porcino, además de que algunos de estos procesos favorecen la obtención de biogás y biofertilizantes. (Hiriart 1984, Caballero-Hernández et al. 2004, López et al. 2008, Moset et al. 2014).

Una de las alternativas que ha resultado factible para el tratamiento del estiércol porcino es el proceso 
de ensilaje (Castellanos-Aceves et al. 2010, GalindoBarboza et al. 2012).

Originalmente el ensilaje se definió como la fermentación láctica de materiales vegetales y fue desarrollado como un método de conservación de forrajes bajo condiciones de anaerobiosis (Hiriart 1984, Muck 2010).

Mediante el ensilaje se controla la actividad microbiana para inducir la fermentación de carbohidratos por las bacterias ácido lácticas (BAL), con o sin el uso de aditivos, lo que favorece las transformaciones bioquímicas para el aprovechamiento de los productos (Hiriart 1984, Muck 2010).

El mayor efecto de conservación de los nutrientes mediante el ensilaje se logra a través de la disminución del $\mathrm{pH}$, que puede reducir los valores hasta 4.5 o 5.0, lo que limita la sobrevivencia de microorganismos competidores de las BAL (Hiriart 1984, Muck 2010).

El proceso de ensilaje no es exclusivo para materiales vegetales, ya que cualquier producto con suficientes carbohidratos fermentables puede ser ensilado (Herrera et al. 2015).

Específicamente, para el tratamiento de estiércol porcino, se ha adecuado el proceso de ensilaje y actualmente se sabe que el producto resultante puede ser aprovechado como ingrediente alternativo de bajo costo en la alimentación de animales (CastellanosAceves et al. 2010).

$\mathrm{Su}$ uso en la suplementación de animales, como vacas en lactación o cerdos en producción, se ha hecho sin mostrar disminución de la productividad o calidad de los productos (Smith et al. 2005, Sahlström et al. 2008, Ruvalcaba et al. 2011a, Ruvalcaba et al. 2011b, Zhao et al. 2013).

Sin embargo, resulta necesario ampliar los esquemas de evaluación para procesos como el ensilaje de excretas, principalmente para generar evidencias científicas sobre la calidad e inocuidad del producto que se obtiene, con la finalidad de hacer más eficiente su aprovechamiento o tratamientos posteriores.

El objetivo del presente estudio fue evaluar el efecto del proceso de ensilaje sobre la reducción de la carga microbiana del estiércol porcino a través del monitoreo de diferentes grupos indicadores de microorganismos y cambios en el $\mathrm{pH}$.

\section{MATERIALES Y MÉTODOS}

\section{Microsilos experimentales}

Para el experimento se colectó estiércol fresco (menos de 24 horas) de cerdos en etapa de crecimiento y desarrollo, de una explotación porcina de tipo intensivo ubicada en el municipio de Tepatitlán de Morelos en Jalisco, el cual se utilizó para preparar los microsilos experimentales.

En el estudio fueron considerados cuatro tratamientos y un grupo testigo; cada tratamiento (Cuadro I) consistió en una combinación de estiércol fresco (85\% para tratamientos T1 y T2; $91.5 \%$ para tratamientos $\mathrm{T} 3 \mathrm{y}$ T4), una fuente de BAL como promotores de la fermentación y un ingrediente con alto contenido de carbohidratos.

CUADRO I. FORMULACIÓN DE LOS SILOS EXPERIMENTALES

\begin{tabular}{lccccc}
\hline & \multicolumn{5}{c}{ Tratamiento } \\
\cline { 2 - 6 } Ingrediente & T1 & T2 & T3 & T4 & Testigo \\
\cline { 2 - 6 } & \multicolumn{5}{c}{ Proporción (\%) } \\
\hline Estiércol de cerdo & 89.5 & 89.5 & 91.5 & 91.5 & 100 \\
Sorgo molido & 10 & 10 & 0 & 0 & 0 \\
Melaza & 0 & 0 & 8 & 8 & 0 \\
Suero de queso & 0.5 & 0 & 0.5 & 0 & 0 \\
Bio-sile & 0 & 0.5 & 0 & 0.5 & 0 \\
\hline
\end{tabular}

${ }^{1}$ Los porcentajes están calculados con base en el peso final del microsilo en base húmeda

El ingrediente rico en carbohidratos correspondió a $10 \%$ peso/peso (p/p) de sorgo para los tratamientos T1 y T2 y, $8 \%$ p/p de melaza con 50 - $60 \%$ de azucares (CONADESUCA 2016) para los tratamientos $\mathrm{T} 3 \mathrm{y} \mathrm{T} 4$.

La fuente de BAL consistió en un inóculo comercial denominado Bio-sile ${ }^{\mathrm{TM}}$ para los tratamientos T2 y T4 $(0.5 \%$ p/p; Lactobacillus plantarum y Pediococcus pentosaceus, $9 \times 10^{9} \mathrm{UFC} / \mathrm{g}$; Hansen, EUA), mientras que la segunda consistió en suero de leche, obtenido como subproducto de la elaboración de queso artesanal (tratamientos T1 y T3, $0.5 \%$ peso/ volumen).

Paralelamente, a partir del suero de leche se recuperaron diferentes aislamientos bacterianos mediante discriminación morfológica colonial en medios de cultivo selectivos para su posterior crioconservación, identificación y caracterización (información no incluida, artículo en proceso).

Como testigo negativo se utilizó estiércol de cerdo sin la adición de una fuente de carbohidratos, ni de bacterias ácido lácticas.

Los microsilos fueron construidos en recipientes de polipropileno estériles de $2.5 \mathrm{~L}$ de capacidad con tapa hermética, en los cuales se depositaron las mezclas correspondientes a cada uno de los tratamientos. 
Para cada tratamiento se prepararon tres microsilos (repeticiones) y se mantuvieron incubados a $25{ }^{\circ} \mathrm{C}$ durante 21 días.

\section{Toma de muestras}

Las muestras del interior de cada uno de los microsilos fueron tomadas diariamente para la determinación del $\mathrm{pH}$ y a los días 0,10 y 21 del tratamiento para la evaluación microbiológica.

Las muestras fueron colectadas en condiciones asépticas mediante el uso de sacabocados estériles para evitar la ruptura del sello superficial del microsilo y las condiciones del mismo.

\section{Cambios en el pH de la excreta}

Para la medición del $\mathrm{pH}$ se tomaron $10 \mathrm{~g}$ de muestra y se diluyeron en $50 \mathrm{~mL}$ de agua destilada estéril. El pH fue determinado a partir del sobrenadante obtenido por gravedad, utilizando un potenciómetro portátil marca Hanna ${ }^{\circledR}$ modelo HI 98127. La medición se llevó a cabo por duplicado (Sadzawka et al. 2005).

\section{Evaluación microbiológica}

Las unidades formadoras de colonia por gramo (UFC/g) de bacterias mesofílicas aerobias (BMA), hongos y levaduras (HyL), enterococos (ENT) y BAL se determinaron a partir de diluciones decimales seriadas de las muestras en agua estéril.

También se determinó la cuenta de organismos coliformes (pruebas presuntiva y confirmativa, OCTP y OCTC respectivamente), organismos coliformes fecales (OCF) y bacterias productoras de ácido sulfhídrico (BPAS) por la técnica del número más probable $(\mathrm{NMP} / \mathrm{g})$. Además, se realizó la búsqueda de cepas patógenas de Escherichia coli y Salmonella spp. (Pérez et al. 2013).

\section{Análisis estadístico}

El diseño experimental consistió en un experimento completamente al azar y los datos obtenidos de los análisis microbiológicos se transformaron a valores base $\log _{10}$. La matriz de datos fue analizada en el paquete estadístico SAS ${ }^{\mathrm{TM}}$ ver. 9.3 (SAS 2012).

Se emplearon los procedimientos de: modelo general lineal (GLM), univariado y análisis de varianza (ANDEVA); se utilizó un nivel de significancia de $\alpha=0.05$ y se complementó el análisis de varianza con la comparación de medias entre tratamientos con la prueba de comparación múltiple de Tukey.

Adicionalmente se efectuaron análisis de asociación (chi cuadrado) y correlación (Pearson) con un nivel de significancia de $\alpha=0.05$ en paquete estadístico SPSS $^{\circledR}$.

\section{RESULTADOS Y DISCUSIÓN}

\section{Disminución del pH}

Uno de los indicadores más simples para evaluar el proceso del ensilaje sobre el valor nutritivo de materiales con alto contenido de humedad es el $\mathrm{pH}$. En general un valor de $\mathrm{pH}$ bajo en ensilados es deseable, debido a que este indica que ha ocurrido una fermentación con producción de ácido láctico (Herrera et al. 2015).

En este estudio, los resultados mostraron que el proceso de ensilaje en todos los tratamientos favoreció la disminución del pH del estiércol (Fig. 1).

Al inicio del proceso los valores de $\mathrm{pH}$ de los tratamientos $\mathrm{T} 3$ y T4 fueron diferentes significativamente $(\mathrm{p}<0.05)$ a los de los tratamientos T1, T2 y el testigo $(5.77 \pm 0.12$ y $5.83 \pm 0.06$ vs $6.93 \pm 0.06$, $7.03 \pm 0.06$ y $6.72 \pm 0.05$, respectivamente).

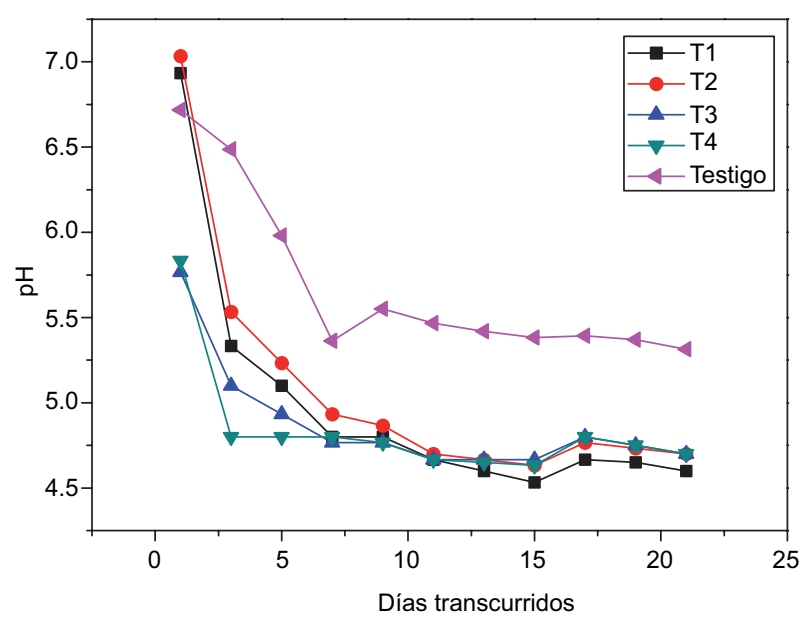

Fig. 1. Valores de $\mathrm{pH}$ durante el proceso de ensilaje de estiércol porcino. T1: Tratamiento 1 (Estiércol+sorgo+suero de quesería); T2: Tratamiento 2 (Estiércol+sorgo+cultivo iniciador); T3: Tratamiento 3 (Estiércol+melaza+suero de quesería); T4: Tratamiento 4 (Estiércol+melaza+cultivo iniciador); Testigo: Estiércol sin aditivos

En general, el pH disminuyó en los primeros 10 días del proceso de ensilaje, con lo que alcanzó valores de $4.8 \pm 0.07$ para los cuatro tratamientos. A partir del onceavo día, la disminución del $\mathrm{pH}$ fue menor.

Al final del experimento (día 21), el pH del grupo testigo se mantuvo superior ( $\mathrm{pH} 5.31 \pm 0.10, \mathrm{p}<0.05$ ) respecto a los demás tratamientos.

Estadísticamente, el tratamiento $\mathrm{T} 1$ fue el que mayor disminución de $\mathrm{pH}$ registró ( $\mathrm{pH}$ final de 4.60 $\pm 0.01)$, lo que marcó una diferencia significativa $(\mathrm{p}<0.05)$ respecto al valor del $\mathrm{pH}$ de los tratamientos $\mathrm{T} 2$, T3 y $\mathrm{T} 4(\mathrm{pH}$ de $4.7 \pm 0.02)$. 
Desde la perspectiva del $\mathrm{pH}$, los niveles alcanzados indican que el proceso fue adecuado, debido a que se ha establecido previamente que un ensilado con humedad superior al $65 \%$ que tiene valores de $\mathrm{pH}$ inferior a 4.8, es considerado un ensilado de buena calidad (Herrera et al. 2015).

\section{Evaluación microbiológica}

Al inicio del estudio todos los tratamientos se encontraron en igualdad de condiciones referente al contenido de bacterias para la mayoría de los grupos indicadores incluidos.

Los grupos en mayor abundancia al inicio del proceso fueron los ENT $\left(8.38 \pm 0.56 \log _{10} \mathrm{UFC} / \mathrm{g}\right)$ y BAL $\left(8.7 \pm 0.22 \log _{10} \mathrm{UFC} / \mathrm{g}\right)$, seguidos por los OCT y OCF $\left(6.7 \pm 0.32 \log _{10} \mathrm{NMP} / \mathrm{g}\right)$, BMA $(6.6 \pm 0.54$ $\left.\log _{10} \mathrm{UFC} / \mathrm{g}\right)$ y $\operatorname{las}$ BPAS $\left(6.19 \pm 0.57 \log _{10} \mathrm{UFC} / \mathrm{g}\right)$ (Fig. 2, Cuadro II).

El indicador en menor proporción al inicio del experimento fue HyL $\left(3.61 \pm 0.46 \log _{10} \mathrm{UFC} / \mathrm{g}\right)$. Sólo se registraron diferencias estadísticas entre tratamientos al inicio del proceso para los contenidos de ENT y BAL $(\mathrm{p}<0.05)$.

Los efectos de la fermentación ácido láctica se observaron desde el día 10 del monitoreo, debido a que en ese tiempo se registraron disminuciones importantes en el contenido de todos los grupos indicadores; en tanto que se mantuvo elevada la proporción de BAL (entre 6.5 y $7.5 \pm 0.31 \log _{10} \mathrm{UFC} / \mathrm{g}$ ).
El mayor efecto de disminución de la carga microbiana se observó al final del proceso de ensilaje (día 21), donde la proporción de OCT y OCF se redujo hasta valores de entre 1 y $2 \log _{10} \mathrm{NMP} / \mathrm{g}$. Mientras que los ENT habían llegado a niveles por debajo del límite de detección de los métodos utilizados.

Al día 21 los contenidos de BMA, HyL, BPAS y BAL presentaron diferencias significativas con respecto al contenido registrado al inicio del proceso de ensilaje $(\mathrm{p}<0.05)$, con disminuciones de entre 1 y $3 \log _{10} \mathrm{UFC} / \mathrm{g}$ para todos los tratamientos. Por su parte, el tratamiento T2 fue el que presentó menor proporción de HyL y BPAS al día 21.

En términos de reducción total del contenido de bacterias, las BMA presentaron el menor nivel de reducción y en promedio quedaron en niveles de entre 4 y $5 \pm 0.89 \log _{10} \mathrm{UFC} / \mathrm{g}$.

No obstante, las BMA no podrían considerarse como determinante de la inocuidad del ensilado, debido a que involucran una gama amplia de géneros bacterianos tanto aerobios como anaerobios facultativos y obligados.

Niveles mayores de reducción fueron observados para OCT y OCF debido a que al final del proceso de ensilaje, los OCT se redujeron a niveles alrededor de los $2 \pm 0.35 \log _{10} \mathrm{NMP} / \mathrm{g}$, sin mostrar diferencias significativas entre tratamientos $(\mathrm{p}>0.05)$.

Por otra parte, para OCF se observó mayor reducción en el tratamiento $\mathrm{T} 1$ en relación al resto de los

CUADRO II. DETERMINACIÓN DE DIFERENTES GRUPOS INDICADORES DE MICROORGANISMOS DURANTE EL ENSILAJE DE EXCRETAS PORCINAS

\begin{tabular}{|c|c|c|c|c|c|c|c|}
\hline \multirow{2}{*}{ Tratamiento } & $\mathrm{BMA}^{1}$ & $\mathrm{HyL}^{1}$ & $\mathrm{OCT}^{2}$ & $\mathrm{OCF}^{2}$ & $\mathrm{ENT}^{1}$ & BPAS $^{2}$ & $\mathrm{BAL}^{1}$ \\
\hline & \multicolumn{7}{|c|}{ Concentración inicial (log10 UFC/g o NMP/g) } \\
\hline $\mathrm{T} 1$ & $6.84^{\mathrm{b}} \pm 0.17$ & $4.36^{\mathrm{a}} \pm 0.32$ & $6.82^{a} \pm 0.38$ & $6.82^{\mathrm{a}} \pm 0.38$ & $8.45^{\mathrm{a}, \mathrm{b}} \pm 0.16$ & $5.78^{\mathrm{a}} \pm 0.51$ & $8.67^{\mathrm{a}, \mathrm{b}} \pm 0.28$ \\
\hline $\mathrm{T} 2$ & $6.29^{b} \pm 0.87$ & $4.20^{\mathrm{a}, \mathrm{b}} \pm 0.17$ & $6.75^{\mathrm{a}} \pm 0.49$ & $6.53^{\mathrm{a}} \pm 0.45$ & $8.59^{\mathrm{a}, \mathrm{b}} \pm 0.38$ & $6.32^{\mathrm{a}} \pm 0.75$ & $8.51^{\mathrm{b}} \pm 0.08$ \\
\hline $\mathrm{T} 3$ & $6.35^{\mathrm{b}} \pm 0.54$ & $4.30^{\mathrm{a}} \pm 1.5$ & $6.57^{\mathrm{a}} \pm 0.16$ & $6.57^{\mathrm{a}} \pm 0.16$ & $7.65^{b} \pm 0.57$ & $5.96^{\mathrm{a}} \pm 0.37$ & $8.70^{\mathrm{a}, \mathrm{b}} \pm 0.09$ \\
\hline $\mathrm{T} 4$ & $6.94^{b} \pm 0.08$ & $4.45^{\mathrm{a}} \pm 0.23$ & $6.92^{\mathrm{a}} \pm 0.22$ & $6.79^{\mathrm{a}} \pm 0.22$ & $8.84^{\mathrm{a}} \pm 0.12$ & $6.70^{\mathrm{a}} \pm 0.33$ & $8.92^{\mathrm{a}} \pm 0.22$ \\
\hline Testigo & $8.71^{\mathrm{a}} \pm 0.25$ & $3.45^{b} \pm 0.05$ & $7.04^{\mathrm{a}} \pm 0.0$ & $7.04^{\mathrm{a}} \pm 0.0$ & $* * *$ & $* * *$ & $7.83^{c} \pm 0.03$ \\
\hline Tratamiento & \multicolumn{7}{|c|}{ Concentración final (log10 UFC/g o NMP/g) } \\
\hline $\mathrm{T} 1$ & $5.95^{\mathrm{a}} \pm 0.75$ & $3.24^{\mathrm{a}} \pm 0.33$ & $2.15^{\mathrm{a}} \pm 0.17$ & $1.25^{b} \pm 0.62$ & $0.00^{\mathrm{a}}$ & $3.63^{\mathrm{b}, \mathrm{c}} \pm 0.0$ & $5.48^{\mathrm{c}} \pm 0.02$ \\
\hline $\mathrm{T} 2$ & $4.94^{\mathrm{a}, \mathrm{b}} \pm 0.17$ & $1.00^{\mathrm{a}} \pm 1.73$ & $1.83^{\mathrm{a}} \pm 0.23$ & $1.52^{\mathrm{a}} \pm 0.05$ & $0.00^{\mathrm{a}}$ & $3.36^{\mathrm{c}} \pm 0.0$ & $5.74^{\mathrm{b}, \mathrm{c}} \pm 0.10$ \\
\hline $\mathrm{T} 3$ & $4.99^{\mathrm{a}, \mathrm{b}} \pm 1.51$ & $1.70^{\mathrm{a}} \pm 2.94$ & $1.52^{\mathrm{a}} \pm 0.05$ & $1.49^{\mathrm{a}} \pm 0.0$ & $0.00^{\mathrm{a}}$ & $4.04^{\mathrm{a}} \pm 0.12$ & $5.93^{\mathrm{a}, \mathrm{b}, \mathrm{c}} \pm 0.01$ \\
\hline $\mathrm{T} 4$ & $4.43^{\mathrm{b}, \mathrm{c}} \pm 0.23$ & $4.66^{\mathrm{a}} \pm 0.15$ & $2.06^{\mathrm{a}} \pm 0.50$ & $1.49^{\mathrm{a}} \pm 0.0$ & $0.00^{\mathrm{a}}$ & $3.86^{\mathrm{a}, \mathrm{b}} \pm 0.19$ & $6.24^{\mathrm{a}} \pm 0.37$ \\
\hline Testigo & $3.56^{\mathrm{c}} \pm 0.03$ & $2.11^{\mathrm{a}} \pm 0.09$ & $1.49^{\mathrm{a}} \pm 0.0$ & $1.49^{\mathrm{a}} \pm 0.0$ & $* * *$ & $* * *$ & $6.04^{\mathrm{a}, \mathrm{b}} \pm 0.13$ \\
\hline
\end{tabular}

Los resultados están expresados en $\log 10$ de ${ }^{1}$ UFC o ${ }^{2}$ NMP de cada uno de los indicadores microbiológicos. ${ }^{\text {a, b }}$ Medias dentro de la misma columna que no comparten una letra son significativamente diferentes $(\mathrm{p}<0.05)$. ${ }^{* *}$ Información no disponible. (OCT, OCF) Organismos coliformes totales y fecales; (H y L) Hongos y Levaduras; (ENT) Enterococos; (BPAS) Bacterias productoras de ácido sulfhídrico; (BMA) Bacterias mesófilas Aerobias; (BAL) Bacterias ácido lácticas 

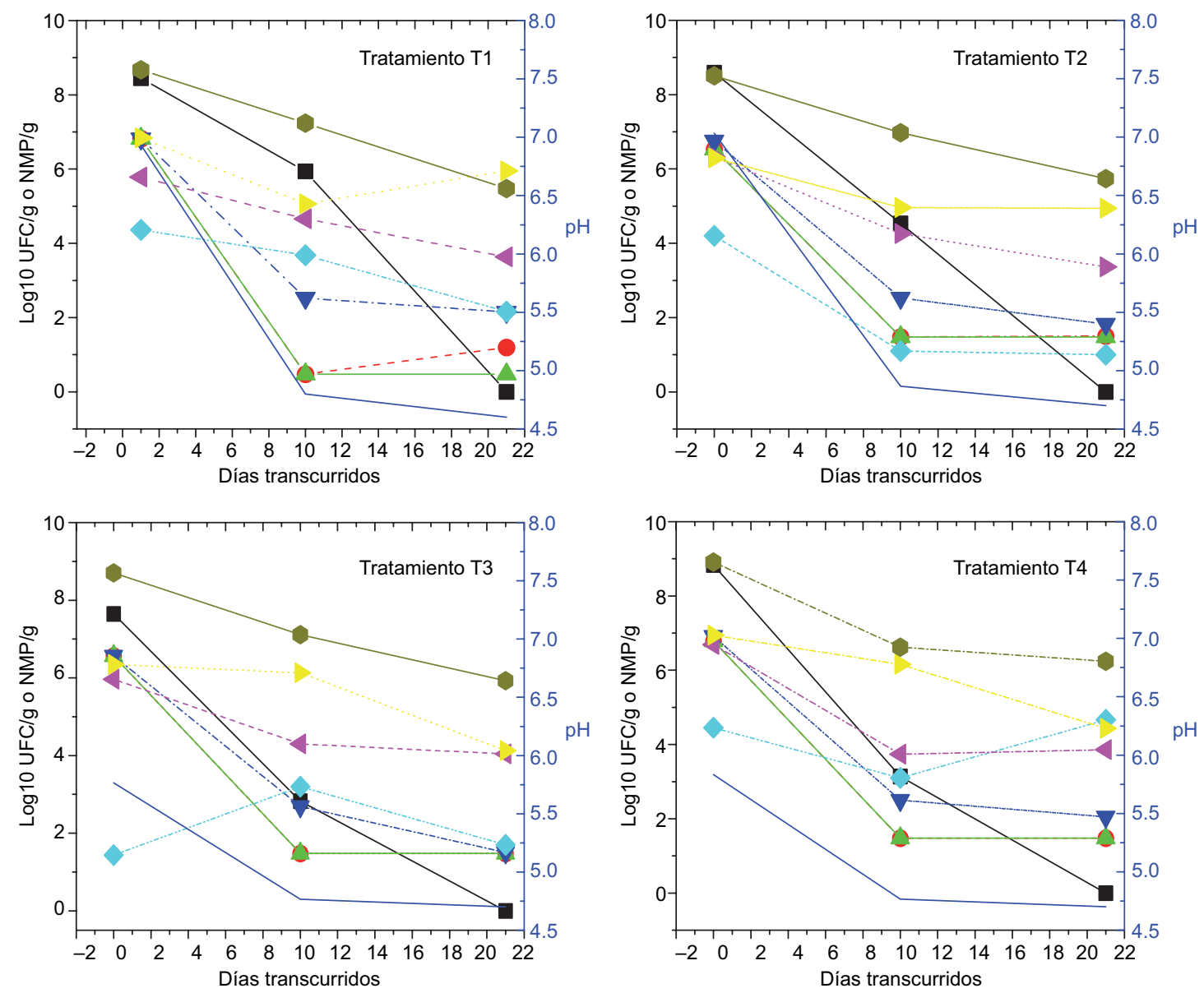

\begin{tabular}{|c|}
\hline- ENT - O--OFC - OCTC $-\nabla$ OCT \\
- HyL -4 - BPAS $>$ BMA - BAL $-\mathrm{pH}$ \\
\hline
\end{tabular}

Fig. 2. Comportamiento microbiológico del ensilado de estiércol porcino con diferentes formulaciones. Tratamiento T1: Estiércol+sorgo+suero de queso; Tratamiento T2: Estiércol+sorgo+cultivo iniciador; Tratamiento T3: Estiércol+melaza+suero de quesería; Tratamiento T4: Estiércol+melaza+cultivo iniciador. (OCT, OCF) Organismos coliformes totales y fecales; (H y L) Hongos y levaduras; (ENT) Enterococos; (BPAS) Bacterias productoras de ácido sulfhídrico; (BMA) Bacterias mesófilas aerobias; (BAL) Bacterias ácido lácticas

tratamientos $(\mathrm{p}<0.05)$, con lo que se disminuyó al nivel de $0.6 \log 10 \mathrm{NMP} / \mathrm{g}$ en comparación con 1.49 $\log 10 \mathrm{NMP} / \mathrm{g}$ para el resto de los tratamientos.

En contraste, las BPAS se mantuvieron presentes hasta el final del proceso, pero con reducciones considerables en las determinaciones. Este grupo de bacterias se redujo más en los tratamientos T1 y T2 en relación a lo determinado para los tratamientos T3 y T4 $(\mathrm{p}<0.05)$.

El medio de cultivo utilizado para BPAS fue medio leche fierro, para determinar el fenómeno "fermentación tormentosa" (fermentación de la leche y fractura del cuajo), que es utilizado como prueba presuntiva para especies del género Clostridium, que está asociado a la producción de olores indeseables característicos del estiércol (Navarro et al. 2009).

Las bacterias del género Clostridium se han reportado como parte de la microbiota del estiércol porcino y se caracterizan por ser anaerobios obligados, esporulados, con crecimientos a temperaturas de entre 30 y $37^{\circ} \mathrm{C}$ a pH de 6.5 a 7 (Zhu 2000), lo que dificulta su eliminación.

Sin embargo, la reducción determinada en el presente estudio para BPAS podría ser un factor clave en la eliminación de malos olores.

Además, la hidrólisis de carbohidratos, proteínas y ácidos grasos asociadas al proceso de fermentación 
ácido láctica (Hansen y Schieberle 2005), se puede relacionar con cambios percibidos en el olor de la excreta durante el proceso de ensilaje, que fue menos desagradable al olfato al final del proceso.

Para confirmar los cambios en las características sensoriales del estiércol, es necesario realizar estudios que incluyan la medición de compuestos asociados al olor, como es el análisis de compuestos volátiles, entre otros.

Es importante mencionar que los niveles de reducción del contenido de bacterias en los tratamientos incluidos son comparables con las reducciones reportadas mediante la aplicación de otro tipo de tratamientos.

Masse et al. (2001), a través de digestión anaerobia a $24{ }^{\circ} \mathrm{C}$, reportan reducciones de entre 2.6 y $2.9 \log _{10}$ tanto para OCF como para Salmonella respectivamente, a partir del día 7 de tratamiento y con reducciones totales a los 28 días.

Sin embargo, los autores de dicha referencia reportan reducciones poco significativas para Clostridium perfringens y Enterococcus spp. con este tratamiento (entre 0 y $0.8 \log _{10}$ ); en contraste con lo obtenido en el presente estudio, donde se observó la eliminación completa de los ENT.

Otro tratamiento comparable en niveles de reducción de cuentas bacterianas en estiércol es la pasteurización.

Sahlström et al. (2008) reportan que para eliminar los enterococos en residuos biológicos utilizados para la producción de biogás, es necesario aplicar pasteurización a $70{ }^{\circ} \mathrm{C}$ durante 30 min, con lo que además se elimina en su totalidad OCT y OCF.

No obstante, a pesar de la intensidad del tratamiento, los autores reportan conteos de más de $4 \log _{10}$ UFC/g para $C$. perfringens posterior a la aplicación del tratamiento.

Los resultados obtenidos en esta investigación también son comparables, e incluso superan a algunos reportes, en los que se evalúa el efecto de la acidificación a temperatura ambiente de estiércol o el uso de biofiltros sobre la reducción del contenido de bacterias (De la Torre et al. 2000, Sahlström et al. 2008, Zhang et al. 2011, McCarthy et al. 2015).

En contraste, sobre la presencia de BAL en todos los tratamientos, no se observó reducción significativa y al día 21 , la cantidad de estos microorganismos se mantuvo en el orden de $\operatorname{los}_{6} \log _{10} \mathrm{UFC} / \mathrm{g}$.

Es importante mencionar que en los tratamientos T3 y T4 se determinaron las mayores concentraciones de BAL al final del proceso, en comparación con los tratamientos T1 y T2 $(\mathrm{p}<0.05)$.
El grupo de las BAL incluye un conjunto amplio de géneros relacionados por sus características tanto morfológicas como fisiológicas, y cuya actividad principal es la producción de ácido como resultado de la fermentación de carbohidratos, por lo que son consideradas como determinantes en los ensilados (Axelsson 2004).

Diversos factores, como el contenido y tipo de carbohidratos y concentración de materia seca, influyen en la prevalencia de las BAL durante la fermentación de los silos, asociado además a las características propias de estas bacterias como la osmotolerancia y su diversidad metabólica (Woolford 1984, McDonald et al. 1991, Oude Elferink et al. 1999).

Lo observado en este estudio coincide con el comportamiento típico de las BAL en ensilados, que incluye un aumento en su concentración durante las etapas iniciales del proceso, para posteriormente disminuir a medida que el $\mathrm{pH}$ desciende y se limita la disponibilidad de nutrientes (Kim et al. 2016, Ni et al. 2017).

Para ensilados de producción se ha reportado que los géneros de BAL predominantes son Lactobacillus, Pediococcus, Leuconostoc, Enterococcus, Lactococcus y Streptococcus que, en su mayoría, son bacterias anaerobias facultativas y mesófilas, con la capacidad de acidificar el medio a niveles de $\mathrm{pH}$ de entre 4 y 5 (Oude et al. 1999).

Adicionalmente, se ha establecido que el proceso de ensilaje de materiales vegetales debe incluir cuatro fases principales diferenciadas: aeróbica, de fermentación, estable y de deterioro (Weinberg y Muck 1996, Merry et al. 1997).

Las tres primeras fases corresponden a una disminución importante en los valores del $\mathrm{pH}$, reducción de indicadores microbiológicos y aumento en la acidez, mientras que en la etapa final se deben mantener los niveles de BAL y de acidez, para poder así realizar la apertura del silo (Weinberg y Muck 1996, Merry et al. 1997).

En el presente estudio fue posible diferenciar las cuatro fases del proceso de ensilaje, a efecto de los cambios en el pH que, a su vez, favorecieron la disminución de los indicadores microbiológicos incluidos.

Referente a la búsqueda de microorganismos patógenos, es importante señalar que en el desarrollo experimental las determinaciones fueron negativas para aislados o cepas patógenas de Salmonella spp. y Escherichia coli.

\section{Asociación de los componentes del ensilado con la disminución de la carga microbiana}

Se determinó el efecto de la fuente de carbohidratos (FC) y la fuente de BAL (FI) en la disminución 
de los diferentes indicadores microbiológicos (Cuadro III).

El descenso del $\mathrm{pH}$ es deseable en los procesos de ensilaje y, en ese contexto, se observó que en los tratamientos con sorgo y suero de leche (tratamiento T1), hubo niveles más bajos de $\mathrm{pH}$ en comparación con lo observado en los tratamientos con melaza o con el cultivo iniciador comercial $(\mathrm{p}<0.05)$.

Específicamente con el uso de sorgo (tratamientos T1 y T2) en contraste con el uso de melaza (tratamientos T3 y T4), se observó una mayor disminución en la cantidad de HyL, debido a que el contenido de este grupo de microorganismos al final del proceso fue menor en los tratamientos con sorgo como fuente de carbohidratos.

El mismo comportamiento en el contenido de $\mathrm{HyL}$ se observó para los tratamientos que incluyeron suero como fuente adicional de BAL, en relación con los que incluyeron el cultivo iniciador.

Por su parte, la FC parece no ser determinante para la reducción o eliminación de OCT, OCF y ENT, debido a que las reducciones observadas para estos indicadores fueron independientes de la FC e incluso de la FI.
En contraste, tanto la FC como la FI tuvieron efecto sobre la reducción de las BPAS, ya que menor contenido de este grupo de bacterias se observó en los tratamientos con sorgo en relación con los que incluyeron melaza.

De igual manera se observó mayor disminución de las BPAS en los tratamientos con suero obtenido de queso en comparación con los que incluyeron el cultivo iniciador comercial $(\mathrm{p}<0.05)$.

Las mayores reducciones de los grupos indicadores fueron observadas en los tratamientos con sorgo como FC respecto a los tratamientos con melaza; este resultado podría estar relacionado con el hecho de que el sorgo podría aumentar el contenido de materia seca en el ensilado y generar una mezcla más homogénea.

A diferencia del uso sorgo, el de melaza, cuya viscosidad la vuelve un material más difícil para su homogeneización durante el preparado, genera mezclas heterogéneas y de difícil manejo (Titterton y Barreba 2001).

Los resultados obtenidos mostraron que el suero proveniente de queso fue mejor como FI en relación al uso de cultivo iniciador para la disminución del $\mathrm{pH}$ y control de algunos indicadores microbiológicos (Cuadro III).

CUADRO III. EFECTO DE LA FUENTE DE CARBOHIDRATOS (FC) Y LA FUENTE DE BAL (FI) SOBRE LA REDUCCIÓN DEL $\mathrm{pH}$ y BACTERIAS DURANTE EL ENSILAJE DE ESTIERCOL PORCINO

\begin{tabular}{|c|c|c|c|c|c|c|c|c|}
\hline & \multicolumn{8}{|c|}{ Indicador microbiológico } \\
\hline & BMA $^{1}$ & $\mathrm{HyL}^{1}$ & $\mathrm{OCT}^{2}$ & $\mathrm{OCF}^{2}$ & $\mathrm{ENT}^{1}$ & BPAS $^{2}$ & BAL $^{1}$ & $\mathrm{pH}$ \\
\hline & \multicolumn{8}{|c|}{ Concentración Inicial (log10 UFC/g o NMP/g) } \\
\hline \multicolumn{9}{|l|}{$\mathrm{FC} / \mathrm{FI}$} \\
\hline Sorgo/Suero (T1) & $6.84^{b} \pm 0.17$ & $4.36^{\mathrm{a}} \pm 0.32$ & $6.82^{\mathrm{a}} \pm 0.38$ & $6.82^{\mathrm{a}} \pm 0.38$ & $8.45^{\mathrm{a}, \mathrm{b}} \pm 0.16$ & $5.78^{\mathrm{a}} \pm 0.51$ & $8.67^{\mathrm{a}, \mathrm{b}} \pm 0.28$ & $6.93^{\mathrm{a}} \pm 0.06$ \\
\hline Sorgo/Biosile (T2) & $6.29^{\mathrm{b}} \pm 0.87$ & $4.20^{\mathrm{a}, \mathrm{b}} \pm 0.17$ & $6.75^{\mathrm{a}} \pm 0.49$ & $6.53^{\mathrm{a}} \pm 0.45$ & $8.59^{\mathrm{a}, \mathrm{b}} \pm 0.38$ & $6.32^{\mathrm{a}} \pm 0.75$ & $8.51^{\mathrm{b}} \pm 0.08$ & $7.03^{\mathrm{a}} \pm 0.06$ \\
\hline Melaza/Suero (T3) & $6.35^{b} \pm 0.54$ & $4.30^{\mathrm{a}} \pm 1.5$ & $6.57^{\mathrm{a}} \pm 0.16$ & $6.57^{\mathrm{a}} \pm 0.16$ & $7.65^{b} \pm 0.57$ & $5.96^{\mathrm{a}} \pm 0.37$ & $8.70^{\mathrm{a}, \mathrm{b}} \pm 0.09$ & $5.83^{\mathrm{b}} \pm 0.12$ \\
\hline Melaza/Biosile (T4) & $6.94^{\mathrm{b}} \pm 0.08$ & $4.45^{\mathrm{a}} \pm 0.23$ & $6.92^{\mathrm{a}} \pm 0.22$ & $6.79^{\mathrm{a}} \pm 0.22$ & $8.84^{\mathrm{a}} \pm 0.12$ & $6.70^{\mathrm{a}} \pm 0.33$ & $8.92^{\mathrm{a}} \pm 0.22$ & $5.83^{\mathrm{b}} \pm 0.06$ \\
\hline Testigo & $8.71^{\mathrm{a}} \pm 0.25$ & $3.45^{b} \pm 0.05$ & $7.04^{\mathrm{a}} \pm 0.0$ & $7.04^{\mathrm{a}} \pm 0.0$ & $* * *$ & $* * *$ & $7.83^{\mathrm{c}} \pm 0.03$ & $6.95^{\mathrm{a}} \pm 0.19$ \\
\hline \multicolumn{9}{|c|}{ Concentración final (log10 UFC/g o NMP/g) } \\
\hline \multicolumn{9}{|l|}{$\mathrm{FC}$} \\
\hline Sorgo $(\mathrm{T} 1, \mathrm{~T} 2)$ & $5.45^{\mathrm{a}} \pm 0.74$ & $1.89^{\mathrm{a}} \pm 1.74$ & $1.99^{\mathrm{a}} \pm 0.25$ & $1.38^{\mathrm{a}} \pm 0.43$ & $0^{\mathrm{a}}$ & $3.48^{\mathrm{b}} \pm 0.15$ & $5.61^{\mathrm{b}} \pm 0.16$ & $4.65^{c} \pm 0.55$ \\
\hline Melaza (T3, T4) & $4.66^{\mathrm{b}} \pm 0.98$ & $2.80^{\mathrm{a}} \pm 2.47$ & $1.77^{\mathrm{a}} \pm 0.44$ & $1.49^{\mathrm{a}} \pm 0.0$ & $0^{\mathrm{a}}$ & $3.95^{\mathrm{a}} \pm 0.18$ & $6.08^{\mathrm{a}} \pm 0.29$ & $4.70^{\mathrm{b}} \pm 0.0$ \\
\hline Testigo & $3.56^{\mathrm{c}} \pm 0.03$ & $2.11^{\mathrm{a}} \pm 0.09$ & $1.49^{\mathrm{a}} \pm 0.0$ & $1.49^{\mathrm{a}} \pm 0.0$ & $* * *$ & $* * *$ & $6.04^{\mathrm{a}, \mathrm{b}} \pm 0.13$ & $5.30^{\mathrm{a}} \pm 0.02$ \\
\hline \multicolumn{9}{|l|}{ FI } \\
\hline Suero $(\mathrm{T} 1, \mathrm{~T} 3)$ & $5.57^{\mathrm{a}} \pm 1.46$ & $1.62^{\mathrm{a}} \pm 2.22$ & $1.82^{\mathrm{a}} \pm 0.37$ & $1.36^{\mathrm{a}} \pm 0.42$ & $0^{\mathrm{a}}$ & $3.84^{\mathrm{a}} \pm 0.23$ & $5.70^{\mathrm{b}} \pm 0.25$ & $4.65^{\mathrm{c}} \pm 0.06$ \\
\hline Bio-sile & $4.67^{b} \pm 0.33$ & $2.83^{\mathrm{a}} \pm 2.29$ & $1.95^{\mathrm{a}} \pm 0.37$ & $1.50^{\mathrm{a}} \pm 0.03$ & $0^{\mathrm{a}}$ & $3.61^{\mathrm{b}} \pm 0.30$ & $5.99^{\mathrm{a}, \mathrm{b}} \pm 0.37$ & $4.70^{\mathrm{b}} \pm 0.0$ \\
\hline Testigo & $3.56^{\mathrm{c}} \pm 0.03$ & $2.11^{\mathrm{a}} \pm 0.09$ & $1.49^{\mathrm{a}} \pm 0.0$ & $1.49^{\mathrm{a}} \pm 0.0$ & $* * *$ & $* * *$ & $6.04^{\mathrm{a}, \mathrm{b}} \pm 0.13$ & $5.30^{\mathrm{a}} \pm 0.02$ \\
\hline
\end{tabular}

Los resultados están expresados en $\log 10$ de ${ }^{1} \mathrm{UFC}$ o ${ }^{2} \mathrm{NMP}$ de cada uno de los indicadores microbiológicos. ${ }^{\mathrm{a}, \mathrm{b}}$ Medias dentro de la misma columna que no comparten una letra son significativamente diferentes $(\mathrm{p}<0.05)$. ${ }^{* * *}$ Información no disponible. (OCT, OCF) Organismos coliformes totales y fecales; (HyL) Hongos y Levaduras; (ENT) Enterococos; (BPAS) Bacterias productoras de ácidosulfhídrico; (BMA) Bacterias mesófilas Aerobias; (BAL) Bacterias ácido lácticas. 
Este resultado podría estar asociado a una mayor diversidad de BAL en el suero, lo que favorecería mayor acumulación de ácidos orgánicos, metabolitos secundarios y sustancias diversas que producen estas bacterias (Parra 2010).

Con respecto al grado de relación entre variables, a través del análisis de chi cuadrado $\left(\mathrm{X}^{2}\right)$, se observaron relaciones significativas $(\mathrm{p}<0.05)$ entre el $\mathrm{pH}$ de los microsilos y el contenido de algunos grupos de microorganismos como HyL, OCF, ENT, BAL y BPAS

De manera similar, a través del análisis de correlación, se corroboró que la disminución de algunos de los grupos indicadores está correlacionada significativamente $(\mathrm{p}<0.05)$ con la disminución del $\mathrm{pH}$, incluido el contenido de BMA $(\rho=0.429)$, OCT $(\rho=0.850)$, OCF $(\rho=0.820)$, ENT $(\rho=0.667)$ y $\operatorname{BPAS}(\rho=0.500)$.

Por lo tanto, se puede inferir que el $\mathrm{pH}$ es una de las variables determinantes para la reducción de la carga microbiana de la excreta porcina.

La disminución del $\mathrm{pH}$ en la excreta se puede relacionar con la presencia de las BAL (principales responsables de la fermentación), que, aunque disminuyeron junto con el $\mathrm{pH}(P=0.514, \mathrm{p}<0.05)$, al final del proceso fue el grupo de mayor abundancia en relación con el resto de los grupos indicadores evaluados.

Los resultados sugieren, por lo tanto, que es importante para el ensilaje de excretas porcinas mantener una abundancia relativa alta de BAL para lograr la reducción del pH y limitar la sobrevivencia de microorganismos no deseados.

\section{CONCLUSIONES}

El uso de bacterias ácido lácticas y una fuente de carbohidratos, como promotores para el establecimiento del ensilaje, resultó una estrategia efectiva para reducir la carga microbiana del estiércol porcino.

Los resultados se asociaron principalmente a la disminución del $\mathrm{pH}$ como factor determinante y que, a su vez, se relacionó directamente con la cantidad de BAL presentes en el proceso, que además se favoreció con la inclusión de sorgo como fuente alterna de carbohidratos.

La disminución de la carga microbiana del estiércol podría contribuir a la descontaminación de las excretas porcinas con fines de inclusión en un esquema de aprovechamiento y manejo integrado de residuos.

\section{REFERENCIAS}

Axelsson L. (2004). Lactic acid bacteria: classification and physiology. En: Lactic acid bacteria: microbiological and functional aspects (S. Salminen, A.V. Wright y A. Ouwehand, Eds.). Marcel Dekker, Nueva York, EUA, pp. 1-66.

DOI: 10.1201/9780824752033.ch1

Borges G.L., Soria F.M. y Ruz F.N. (2003). Contenido de macronutrimentos en sustratos de bagazo de henequén y excreta porcina y su efecto en el desarrollo de plántulas de papaya. Revista Chapingo serie Horticultura 9 (2), 291-304.

Bórquez J.L., González M.S.S., Pinos R.J.M., Domínguez I. y Bárcena J.R. (2009). Feeding value of ensiling fresh cattle manure with molasses or bakery by-products in lambs. Livest. Sci. 122 (2-3), 276-280. DOI: 10.1016/j.livsci.2008.09.009

Bulkowska K., Pokoj T., Klimiuk E. y Gusiatin Z.M. (2012). Optimization of anaerobic digestion of a mixture of Zea mays and Miscanthus sacchariflorus silages with various pig manure dosages. Bioresour. Technol. 125, 208-216. DOI: 10.1016/j.biortech.2012.08.078

Caballero H.A.I., Castrejón P.F., Martínez G.R., Ángeles C.S., Pérez R.M. y Buntinx S.E. (2004). Survival and viability of Ascaris suum and Oesophagostomum dentatum in ensiled swine faeces. Bioresour. Technol. 94 (2), 137-142.

DOI: 10.1016/j.biortech.2003.12.008

Castellanos A.A., Salazar G.G., Hernández M.P., Domínguez A.G. y Barrera C.G. (2010). Uso de ensilado de cerdaza en la alimentación animal. Folleto para productores. Instituto Nacional de Investigaciones Forestales, Agrícolas y Pecuarias, Tepatitlán de Morelos, Jalisco, México, $21 \mathrm{pp}$.

CONADESUCA (2016). Melazas de caña de azúcar y su uso en la fabricación de dietas para ganado. Consejo Nacional para el Desarrollo Sustentable de la Caña de Azúcar. Nota informativa, México, D. F., México, 9 pp.

de la Torre A.I., Jiménez J.A., Carballo M., Fernandez C., Roset J. y Muñoz M.J. (2000). Ecotoxicological evaluation of pig slurry. Chemosphere 41 (10), 1629-1635. DOI: $10.1016 / \mathrm{S} 0045-6535(00) 00038-2$

FAO (2013a). Greenhouse gas emissions from pig and chicken supply chains - A global life cycle assessment. Organización de las Naciones Unidas para la Alimentación y la Agricultura, Roma, Italia. [en línea] http://www. fao.org/docrep/018/i3460e/i3460e00.htm 06/04/2017

FAO (2013b). Greenhouse gas emissions from ruminant supply chains - A global life cycle assessment. Organización de las Naciones Unidas para la Alimentación y la Agricultura, Roma, Italia. [en línea] http://www. fao.org/docrep/018/i3461e/i3461e00.htm 06/04/2017 
Galindo B.A.J., Domínguez A.G., Salazar G.G., Sánchez G.F.J. y Avalos C.M.A. (2012). Uso de ensilado de cerdaza en la alimentación animal. Memorias. Vamos al campo 2012. Tepatitlán de Morelos, Jalisco, México, 19 de octubre, 2012, pp. 57-62.

García A., León R., Míreles S., Castro J.P., García A.A., Roa J.J. y Guerrero L.A. (2010). Contaminación ambiental en explotaciones porcinas mexicanas e incumplimiento de la norma ambiental. Revista Computadorizada de Producción Porcina 17 (3), 243-246.

Hansen A. y Schieberle P. (2005). Generation of aroma compounds during sourdough fermentation: applied and fundamental aspects. Trends Food Sci. Technol. $16(1-3), 85-94$.

DOI: $10.1016 /$ j.tifs. 2004.03 .007

Herrera C.J., Naranjo J.N., Almaraz A.N., Uribe S.N., y Rosas M.I. (2015). El ensilado. Proceso, manipulación y uso del ensilaje. Instituto Politécnico Nacional, México, D. F., México, 114 pp.

Hiriart L.M. (1984). Ensilaje, composición química, calidad fermentativa, valor nutritivo. Investigación y progreso agropecuario. Carillanca 3 (1-4), 28-30.

Kim J.S., Lee Y.H., Kim Y.I., Ahmadi F., Oh Y.K., Park J.M. y Kwak W.S. (2016). Effect of microbial inoculant or molasses in fermentative quality and aerobic stability of sawdust-based spent mushroom substrate. Bioresour. Technol. 216, 188-195.

DOI: 10.1016/j.biortech.2016.05.056

López Y., Arece J., León E., Aróstica N. y Ojeda F. (2008). Efecto de la inclusión de un ensilaje mixto en el comportamiento productivo de ovejas pelibuey en pastoreo. Pastos y Forrajes 31 (1), 73-82.

Martínez V., García M.D. y Ly J. (2004). Estimados de excreción fecal de cerdos como material de ingreso en biodigestor y para composta. Revista Computadorizada de Producción Porcina 11, 283-289.

Masse D., Gilbert Y. y Topp E. (2011). Pathogen removal in farm-scale psychrophilic anaerobic digesters processing swine manure. Bioresour. Technol. 102 (2), 641-646.

DOI: 10.1016/j.biortech.2010.08.020

McCarthy G., Lawlor P.G., Carney K.N., Zhan X., Gutierrez M. y Gardiner G.E. (2015). An investigation into the removal of Salmonella and enteric indicator bacteria from the separated liquid fraction of raw or anaerobically digested pig manure using novel on-farm woodchip biofilters. Sci. Total. Environ. 514, 140-146. DOI: $10.1016 /$ j.scitotenv.2014.12.036

McDonald P., Henderson A.R. y Heron S.J.E. (1991). The biochemistry of silage. 2 ed. Chalcombe Publications, Marlow, Bucks, Reino Unido, 340 pp.

Merry R.J., Lowes K.F. y Winters A. (1997). Current and future approaches to biocontrol in silage. Memorias. 8th Int. Symposium forage conservation, Brno. Research Institute of Animal Nutrition Ltd., Pohorelice, República Checa, 21 de septiembre al 1 de octubre, 1997, pp. 17-27.

Moset V., Bertolini E., Cerisuelo A., Cambra M., Olmos A. y Cambra-López M. (2014). Start-up strategies for thermophilic anaerobic digestion of pig manure. Energy 74, 389-395. DOI: 10.1016/j.energy.2014.07.003

Muck R.E. (2010). Silage microbiology and its control through aditives. Revista Brasileira de Zootecnia 39, 183-191. DOI: 10.1590/S1516-35982010001300021

Navarro H.V., Olea R.M.A. y Torres V.M.R. (2009). Clostridium perfringens en alimentos cárnicos. Universidad de Guadalajara, Guadalajara, Jalisco, México, 176 pp.

Ni K., Wang F., Zhu B., Yang J., Zhou G., Pan Y. y Zhong J. (2017). Effects of lactic acid bacteria and molasses additives on the microbial community and fermentation quality of soybean silage. Bioresour. Technol. 238, 706-715. DOI: 10.1016/j.biortech.2017.04.055

Oude S.J.W.H., Driehuis F., Gottschal J.C. y Spoelstra S.F. (1999). Silage fermentation processes and their manipulation. Memorias. Organización de las Naciones Unidas para la Alimentación y la Agricultura. Electronic conference on tropical silage. 1 de septiembre al 15 de diciembre, 1999. [en línea] http://www.fao. org/docrep/005/X8486E/x8486e09.htm 20/02/2017

Parra H.R.A. (2010). Review. Bacterias ácido lácticas: papel funcional en los alimentos. Rev. Bio. Agro 8 (1), 93-105.

Pérez U.M., Amaro R.A. y Castro C.N. (2013). Manual integrado de la normatividad y métodos de detección y medición de organismos microbiológicos en alimentos como vegetales y frutas frescas, productos del mar y cárnicos de animales terrestres. Centro Nacional de Metrología, El Marqués, Querétaro, México, 244 pp.

Philippe F.X. y Nicks B. (2015). Review on greenhouse gas emissions from pig houses: production of carbon dioxide, methane and nitrous oxide by animals and manure. Agr. Ecosyst. Environ. 199, 10-25. DOI: 10.1016/j.agee.2014.08.015

Ruvalcaba G.J.M., Domínguez A.G., Salazar G.G., Arias C.L.E., Castellanos A.A., Hernández M.P., Romero J.J.H. y Ruiz A.C.R. (2011a). Uso de ensilado de cerdaza en la alimentación de vacas en lactación y su efecto sobre el perfil de ácidos grasos de la leche. Memorias. XLVII Reunión Nacional de Investigación Pecuaria, León, Guanajuato, México. 12 al 14 de octubre, 2011, pp. 184.

Ruvalcaba G.J.M., Domínguez A.G., Salazar G.G., Arias C.L.E., Hernández M.P., Castellanos A.A. y Romero J.J.H. (2011b). Uso de ensilado de cerdaza en la alimentación de vacas lecheras y su efecto sobre las características higiénico-sanitarias de la leche. Memorias. 
XIII Congreso Internacional Inocuidad de Alimentos. Universidad de Guadalajara, Puerto Vallarta, Jalisco, México. 3 al 5 de noviembre, 2011, pp. 113-116.

Sadzawka R. A., Carrasco R. M. A., Grez Z. R. y Mora G. M.L. (2005). Métodos de análisis de compost. Instituto de Investigaciones Agropecuarias, Serie Actas No 30, Santiago, Chile, 142 pp.

Sahlström L., Bagge E., Emmoth E., Holmqvist A., Danielsson-Tham M.L. y Albihn A. (2008). A laboratory study of survival of selected microorganisms after heat treatment of biowaste used in biogas plants. Bioresour. Technol. 99 (16), 7859-7865. DOI: 10.1016/j. biortech.2007.09.071

Sánchez M. y González J.L. (2005). The fertilizer value of pig slurry. I. Values depending on the type of operation. Bioresour. Technol. 96 (10), 1117-1123. DOI: 10.1016/j.biortech.2004.10.002

SIAP (2014). Resumen de la producción pecuaria en México. Servicio de Información Agroalimentaria y Pesquera [en línea]. https://www.gob.mx/siap 28/06/15

Smith S.R., Lang N.L., Cheung K.H. y Spanoudaki K. (2005). Factors controlling pathogen destruction during anaerobic digestion of biowastes. Waste Manag. 25 (4), 417-425. DOI: 10.1016/j.wasman.2005.02.010

Steinfeld H., Gerber P., Wassenaar T., Castel V., Rosales C. y de Haan C. (2006). Livestock's long shadow: environmental issues and options. Organización de las Naciones Unidas para la Alimentación y la Agricultura. [en línea] http://www.fao.org/docrep/010/a0701e/ a0701e00.HTM 20/01/2017
Titterton M. y Barreba F.B. (2001). Ensilaje de gramíneas y leguminosas en los trópicos. Memorias. Uso de ensilaje en el trópico privilegiando opciones para pequeños campesinos. Organización de las Naciones Unidas para la Alimentación y la Agricultura. Conferencia electrónica sobre el ensilaje en los trópicos. 1 de septiembre al 15 de diciembre, 1999 [en línea]. http://www.fao.org/docrep/005/X8486S/x8486s06. htm 20/01/2017

Weinberg Z.G. y Muck R.E. (1996). New trends and opportunities in the development and use of inoculants for silage. FEMS Microbiol. Rev. 19 (1), 53-68. DOI: 10.1016/0168-6445(96)00025-3

Woolford M.K. (1984). The silage fermentation. Vol. 14. Microbiological series. Marcel Dekker, Inc., Nueva York, EUA, 350 pp.

Zhang D., Yuan X., Guo P., Suo Y., Wang X., Wang W. y Cui Z. (2011). Microbial population dynamics and changes in main nutrients during the acidification process of pig manures. J. Environ. Sci. 23 (3), 497-505. DOI: 10.1016/S1001-0742(10)60434-2

Zhao H.Y., Li J., Liu J.J., Lü Y.C., Wang X.F. y Cui Z. (2013). Microbial community dynamics during biogas slurry and cow manure compost. J. Integr. Agr. 12 (6), 1087-1097. DOI: 10.1016/S2095-3119(13)60488-8

Zhu J. (2000). A review of microbiology in swine manure odor control. Agric. Ecosyst. Environ. 78, 93-106. DOI: 10.1016/S0167-8809(99)00116-4 